\title{
P I 4- I 5 LB. The safety and immunogenicity of HIV-I vaccines based on DNA and replication competent vaccinia vector in phase I
} clinical trial

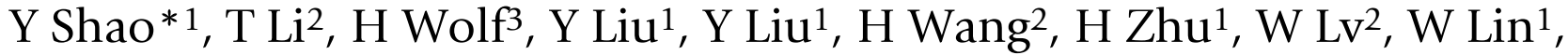 \\ J Chen ${ }^{1}$, H Liang ${ }^{1}, \mathrm{~K}_{\text {Hong }}{ }^{1}$ and $\mathrm{M} \mathrm{Jia}^{1}$
}

Address: ${ }^{1}$ National Center for HIV/STD Prevention and Control, China CDC, Beijing, PR China, ${ }^{2}$ Dept. Infectious Disease, Peking Union Medical College Hospital, Beijing, PR China and ${ }^{3}$ Institute of Medical Microbiology, Regensburg University, Regensburg, Germany

* Corresponding author

from AIDS Vaccine 2009

Paris, France. 19-22 October 2009

Published: 22 October 2009

Retrovirology 2009, 6(Suppl 3):P404 doi:10.1 I86/I742-4690-6-S3-P404

This abstract is available from: http://www.retrovirology.com/content/6/S3/P404

(c) 2009 Shao et al; licensee BioMed Central Ltd.

\section{Background}

To assess the safety and immunogenicity of recombinant HIV vaccines of DNA and replicating competent Tiantan vaccinia (rTV)vector, the small pox vaccine used in China.

\section{Methods}

HIV-1 CN54 gag, pol and env genes were constructed into DNA and rTV vectors. 48 healthy participants were either inoculated with rTV (5 × 104 pfu, skin scratches $)$ or DNA vaccine ( $2 \mathrm{mg}, 4 \mathrm{mg}$, i.m.) alone, in combination, or placed on the placebo. The participants were monitored up to 36 weeks for clinical symptoms and laboratory tests. Vaccine induced immunogenicity were measured by ELIspot, ICS, and antibody assays.

\section{Results}

Typical skin reaction in all rTV vaccinated 24 subjects, enlargement of the lymph nodes under the same arm receiving rTV ( 12 cases) and slight fever (37.2 for 1 day, 1 case) were observed. No severe adverse events related to the vaccines were found. In rTV single vaccination group, positive IFN-g ELIspot was detected only in 6 of 7 vaccinia naïve subjects. In DNA/rTV group, $\mathrm{T}$ cell responses were detected in 15/16 (IFN-g ELIspot) and 16/16 (ICS) of vaccinia naïve and 5/6 (IFN-g Elispot) and 5/6 (ICS) of vaccinia experienced. The responses of CD4 cells were higher than those of CD8 cells (100\% versus 44\%) and responsive rates to IL-2 and IFN-g were similar. HIV-1 gag and env antibodies were detected only in the DNA priming and rTV boost groups and mainly among vaccinia naïve people (13/16 in naïve and $1 / 6$ in experienced). Both $\mathrm{T}$ cell and antibody responses maintain by the end of the study at week 36 .

\section{Conclusion}

The vaccines are well tolerated and safe. It can stimulate HIV-1 specific T cell response in a single rTV vaccination and both $\mathrm{T}$ cell and antibody responses in DNA prime rTV boost. The vaccines are currently moving to phase II clinical trial in China. 